\title{
Conceptual Replicability
}

Matthew B.A. McDermott, Shirly Wang, Nikki Marinsek, Rajesh Ranganath, Marzyeh Ghassemi, Luca Foschini

\section{Source}

Matthew B. A. McDermott, Shirly Wang, Nikki Marinsek, Rajesh Ranganath, Marzyeh

Ghassemi, Luca Foschini. (2019). Reproducibility in Machine Learning for Health.

arXiv:1907.01463v1 [Cs.LG]. https://arxiv.org/pdf/1907.01463.pdf

It describes the notion of how well the desired results reproduces under conditions that mach the conceptual description of the purported effect. 